\title{
Discontinuation of pediatric trials
}

It is said that a research study is completed when its results are submitted for consideration to the scientific community, which will ultimately assess, accept, and eventually adopt those results.

In order for our peers to determine the validity of a scientific study, they need to get all the information about the study to adequately review its methodological rigor and the significance of its results. Although the most frequent form of disseminating information about study results is to present them at scientific events, the information necessary to fully evaluate a research work requires the publication in full text.

Unfortunately, the rate of full-text publication is low. In a society where scholarly work is highly recognized, this may be serious; however, in our setting, even some of the different professional assessment systems offer little consideration to scientific publication.

When described this way, at first glance, it seems that the fact of not publishing a research study would only affect authors and, eventually, the scientific development of the country. Nevertheless, non-publication may be detrimental to other stakeholders involved: funding entities (overt and hidden), institutions (which are deprived of visibility and recognition) and, more importantly, research subjects, who ran risks and contributed their time and commitment.

There is enough evidence on the nonpublication of full-text versions of abstracts corresponding to completed studies presented at scientific events. ${ }^{1}$ However, failure to disseminate research findings may start at an earlier stage, when research studies are interrupted before being completed. Such practice is particularly serious in the case of children given the additional difficulties entailed by research studies in pediatrics.

Pica and Bourgeois ${ }^{2}$ pointed out that this situation affects almost one fifth of controlled clinical trials conducted in the pediatric population and registered in ClinicalTrials.gov, the registry and results database of the National Institutes of Health (NIH) of the United States. Out of 559 registered pediatric studies, $19 \%$ were discontinued early; and of the 455 completed studies, 29.8\% remained unpublished.

The requirement for advanced registration of controlled clinical trials as a method for publication in most scientific journals worldwide ${ }^{3}$ was of vital importance to improve research visibility and reduce the risk concerning the non-publication of negative outcomes. However, this policy is not mandatory for observational studies yet. ${ }^{4}$ For this reason, it is difficult to estimate the true magnitude of the problem caused by non-publication and discontinuation of studies, and such phenomenon may possibly be far more significant than what has been mentioned here.

Pica and Bourgeois have found that an important determinant for trial discontinuation is not reaching the sample size and not being funded by the pharmaceutical industry. Although it may call the attention that being funded by industry is associated with non-publication at 36 months, it should be considered that studies funded by the pharmaceutical industry required more time to be published than those that were not (33 versus 24 months).

Regardless of all the reasons possibly involved in the discontinuation and/or non-publication of a trial (funding, type of intervention, logistical problems, failure to reach sample size, etc.), it is quite likely that the lack of adequate planning is the true cause in many of these cases. Once again, emphasis should be placed on establishing realistic working protocols, with strict sample sizes and considering the worst-case scenarios for trials. Only in this way it will be reasonably possible to conclude a study and have results published.

Although rates of discontinuation $(19 \%)$ and non-publication $(29.8 \%)$ observed in pediatrics by Pica are somewhat more encouraging than those reported by Kasenda et al. ${ }^{5}$ for all registered studies (25\% and $33 \%$, respectively), mostly in the adult population, it must not be forgotten that the investigator is committed to his or her patients, research subjects who trust him or her, trust which is even stronger in the case of children. Out of all those ethical considerations in relation to trial discontinuation, ${ }^{6}$ probably the most important one is that of not betraying the confidence of those who agreed to participate in the study with the altruistic expectation of collaborating with science.

Fernando Ferrero, M.D. Hospital General de Niños Pedro de Elizalde

http:/ / dx.doi.org/10.5546/aap.2017.eng.308

To cite: Ferrero F. Discontinuation of research in pediatrics. Arch Argent Pediatr 2017;115(4):308-309.

\section{REFERENCES}

1. Scherer RW, Langenberg P, Von Elm E. Full publication of results initially presented in abstracts. Cochrane Database Syst 
Rev 2007;(2):MR000005.

2. Pica N, Bourgeois F. Discontinuation and nonpublication of randomized clinical trials conducted in children. Pediatrics 2016;138(3):e20160223.

3. De Angelis CD, Drazen JM, Frizelle FA, et al. Clinical trial registration: a statement from the International Committee of Medical Journal Editors. JAMA 2004;292(11):1363-4.

4. Dal-RéR, IoannidisJP, Bracken MB, etal. Making prospective registration of observational research a reality. Sci Transl
Med 2014;6(224):224cm1.

5. Kasenda B, VonElmE, You J, etal.Prevalence, characteristics, and publication of discontinued randomized trials. JAMA 2014;311(10):1045-51.

6. Kasenda B, Von Elm EB, You J, et al. Learning from failure-rationale and design for a study about discontinuation of randomized trials (DISCO study). BMC Med Res Methodol 2012;12:131.

\section{The principle of synchronicity-equivalence and chronomedicine: relevance and methodological exploration}

\section{INTRODUCTION}

More than half a century ago, Carl Jung and Wolfgang Pauli developed a revolutionary hypothesis: natural phenomena are ruled not only by the principle of causality, but also by a kind of natural order that interconnects and regulates all events. Jung designated it as "principle of synchronicity or equivalence" (SE), according to whether or not it was perceived by the human mind. ${ }^{1}$ Currently, the effect of the principle of SE on the natural world is known as "chronobiology". The effect of the principle of SE on health and disease processes is known as "chronomedicine". Modern research methods allow for the identification of organization patterns that, in spite of their importance, usually go unnoticed by the human mind.

This paper briefly describes the origin, evolution and significance of the concept of SE in modern medicine. ${ }^{2,3}$

\section{Causality: the familiar principle}

Causality consists of a successive series of events in which the precedent (cause) gives rise to the subsequent (effect), within the confines of time, space and formal logic. ${ }^{1,2}$ However, causality is actually a habit of the mind, born from a repeated historical precedent originating an abstraction (bias). This abstraction results from a mixture of habits, beliefs and common sense, since every phenomenon does not stem from a single cause, but from a network of causes that our lack of subtlety prevents us from perceiving in its entirety. ${ }^{2}$ Therefore, causality is only applicable to certain portions of reality, and not to reality as a whole or unicity; from this latter perspective, everything is, to a certain extent, the cause of everything (unus mundus). ${ }^{2}$

\section{Synchronicity-equivalence: the new principle}

Synchronicity consists in the detection, by the human mind, of a meaningful and a causal coincidence between two or more events in the world. For this reason, this principle is also known as the "meaningful coincidence" or "a causal connection", because the relationship established between the events in question is not cause-effect nor random; their relation exceeds the likelihood to be attributed to chance. ${ }^{1}$ Beyond the significance of synchronicity as a psychological phenomenon, ${ }^{4}$ that is, as the intuitive capacity of the human mind to perceive the relation between two events significantly but not causally related, its value is based on evidencing the existence of a principle of nature. ${ }^{5}$

The perception of this connection is not an invention of the mind, but the brief and intuitive perception of the triggering of a natural pattern that overlays, at that moment, several phenomena. These are oscillatory patterns which -even though omnipresent-, usually go unnoticed to a human being, who can only catch glimpses of them (synchronic episodes). Therefore, Jung established a difference between the existence of organization patterns regardless of their perception by man: the principle of equivalence (Gleichartigkeit); and the perception of patterns by the human mind: principle of synchronicity (Sinngemässe Koinzidenz). ${ }^{5}$

It has been proposed that the perception of these patterns (equivalence) in a conscious fashion (synchronicity) would be possible thanks to the presence of certain reflexes located in areas that are phylogenetically old in the central nervous system (autonomous-reptilian nervous system). These areas are responsible for aligning (consciously or unconsciously) the body to world rhythms. This would explain the existence of synchronic events in human beings, as well as the collective coordinated behaviors of several living creatures, both among them and in compass with the cycles of Earth. ${ }^{1,4,6,7}$ 